Background: The autoimmune regulator gene ( $A I R E)$ has very important role in self-tolerance. Estrogen induced hypermethylation of $A I R E$ promoter was put forward as one of the reasons for susceptibility to autoimmunity in females. [1] Sulphasalazine is a commonly used drug in rheumatology and is known to be responsible for various autoimmune side effects.

Objectives: We aimed to investigate the effects of commonly used treatments on the methylation status of AIRE and levels of interleukin-16 (IL16), interleukin-1 $\beta$ (IL1 $\beta$ ), and interferon- $\gamma$ (IFN $\gamma$ ), in lipopolysaccharides (LPS)-induced RAW264.7 macrophage cells which mimic inflammatory state in rheumatoid arthritis in vitro.[2] Methods: RAW264.7 cells were stimulated by LPS $(1 \mu / \mathrm{mL})$. Cell viability test was performed to determine drug concentrations. Following drug treatments, cell media was isolated for the determination of IL16, IL1 $\beta$, and IFN $\gamma$ levels by ELISA. Also, the cells detached by trypsin-EDTA were used for DNA isolation and bisulfite modification. Then, the promoter methylation status of AIRE was analyzed with methylation-specific PCR and agarose jel electrophoresis.

Results: Our results demonstrated that the AIRE promoter is highly methylated in absence of any inflammatory stimulus.LPS treatment changed methylation status to unmethylated form. Leflunomide(LEF), sulfasalazine(SLZ) and methotrexate(MTX) significantly altered methylation status as the methylated $(p=0.041, p<0.001, p<0.001$, respectively). Surprisingly, SLZ treatment caused significantly more methylation in AIRE promoter than other drugs $(p<0.001$,Fig 1A). Analysis of IL16, IL1 $\beta$ and IFN $\gamma$ levels demonstrated that LPS caused potent increase in all three cytokines $(p<0.001$, Fig 1B-D). LEF and SLZ significantly prevented LPS-induced increase in these cytokines $(p=0.025, p<0.001$ for IL-16, $\mathrm{p}<0.001$ for IL-1 $\beta$ and IFN $\gamma$, Fig 1B-D). Although MTX exacerbated LPS-induced increase in IL16 levels, it inhibited LPS induced increase in IL1 $\beta$ and IFN $\gamma$ levels $(p<0.001$, Fig 1B-D).

A
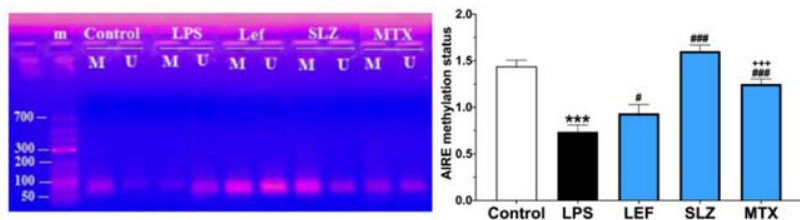

B

C

D

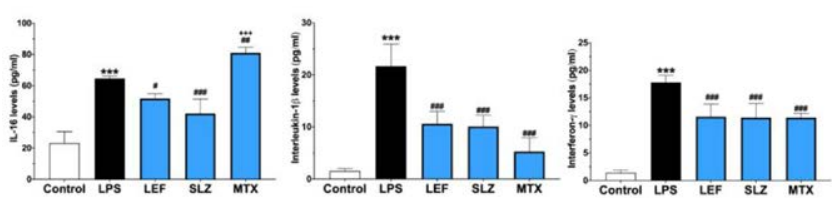

Figure 1. PCR gel image. All data expressed as mean \pm SD. ${ }^{\star * *} p<0.001$ versus control, $\# \# \#<0.001, \# p<0.05$ versus LPS, $+++p<0.001$ versus sulfasalazine.

Conclusion: Our results suggest that although LEF,SLZ and MTX potently suppresses LPS-induced inflammatory cytokines, they affect AIRE methylation differently.Changes in the methylation status of $A I R E$ are important for autoimmunity. Relative increase in methylation of $A I R E$ promoter by $S L Z$ usage can be responsible for its autoimmune side effects, like drug induced lupus or hypersensitivity reactions, which are not common in MTX and LEF usage.

IL16 is a key regulator of biological properties of CD4+T cells, it also regulates migration of CD4+CD25+Treg cells.[3] MTX treatment was shown to increase Treg cells in early rheumatoid arthritis patients. LEF and SLZ's effect on Treg cells was shown to be different from that of MTX.[4] Our results demonstrated that MTX exacerbated LPS-induced increase in IL16 levels in contrary to LEF and SLZ. This difference may also be responsible for the different effect of these medications on Treg functions.

The different effect of commonly used disease modifying drugs on IL16 levels and methylation of $A I R E$ promoter is interesting and deserves attention.

REFERENCES:

[1] Berrih-Aknin, Le Panse R, Dragin N.AIRE:a missing link to explain female susceptibility to autoimmune diseases. Ann.N.Y.Acad.Sci, 2018;1412(1):21-32.

[2] Lin $\mathrm{Y}-\mathrm{Y}$, Jean $\mathrm{Y}-\mathrm{H}$, Lee $\mathrm{H}-\mathrm{P}$ et al. Excavatolide $\mathrm{B}$ attenuates rheumatoid arthritis through the inhibition of osteoclastogenesis. Marine Drugs, 2017; 15(1):9.

[3] Skundric DS, Cruikshank WW, Montgomery PC et al. Emerging role of IL-16 in cytokine-mediated regulation of multiple sclerosis. Cytokine, 2015;75(2):234-248.

[4] Oh JS, Kim Y-G, Lee SG et al. The effect of various disease modifying anti-rheumatic drugs on suppressive function of CD4+CD25+ regulatory Tcells. Rhematol Int. 2013;33:381-388

Disclosure of Interests: None declared

DOI: 10.1136/annrheumdis-2021-eular.3335

\section{AB0013 ASSOCIATION BETWEEN STAT4 POLYMORPHISM AND MANIFESTATIONS OF SLE}

V. Agarwall ${ }^{1}$, S. Kakati ${ }^{1}$, P. Debbaruah ${ }^{2} .{ }^{1}$ Assam Medical College and Hospital, Medicine, Dibrugarh, India; ${ }^{2}$ Assam Medical College and Hospital, Anatomy, Dibrugarh, India

Background: SNP rs7574865, located within the third intron of STAT4 gene at chromosome 2, has been associated with susceptibility to SLE among different ethnic groups. ${ }^{1,2}$ Interestingly, we recently have documented an association between this gene and susceptibility to systemic lupus erythematosus (SLE) in Indian population. ${ }^{3}$

Objectives: To determine whether the STAT4 (rs7574865) SNP is associated with clinical and immunological manifestations in SLE.

Methods: The study was carried out on 100 unrelated SLE (SLICC criteria 2012) patients from North-East India. Genotyping of STAT4 rs7574865 SNP was done using Taqman probe and Real-Time Polymerase chain reaction. An association study was performed between the alleles and genotypes of STAT4 rs7574865 with the clinical and immunological manifestations included in the SLE SLICC classification criteria. For all analysis, the statistical significance was fixed at $5 \%$ level of significance $(\mathrm{p}<0.05)$.

Results: The mean duration of illness was $2.69 \pm 2.55$ years. Cases and Controls remained in Hardy-Weinberg equilibrium. The occurrence of Photosensitivity and hyperpigmentation was significantly higher in TT genotype group (97.22\% and $77.77 \%$, respectively) with $\mathrm{p}<0.001$ in each case.SLE patients with nephritis (Albuminuria $>500 \mathrm{mg} / 24$ hours) and elevated serum creatinine were both significantly higher in TT genotype group as compared to GT and GG $(p<0.001$ and $\mathrm{p}=0.001$ respectively). The Anti-dsDNA antibody was significantly associated with TT genotype $(p<0.001)$.

Conclusion: Our study provides evidence regarding the association between STAT4 rs7574865 gene polymorphism is risk factor for cutaneous manifestations, Lupus nephritis and Anti ds-DNA positivity in SLE. So, our findings reinforce the need for further association studies including prospective studies with larger subjects in order to replicate such findings.

\section{REFERENCES:}

[1] Graham RR, Ph D, Hom G, Ph D, Behrens TW, Bakker PIW De, et al. and the Risk of Rheumatoid Arthritis and Systemic Lupus Erythematosus. N Engl J Med. 2007;357(10):977-86.

[2] Yuan H, Feng JB, Pan HF, Qiu LX, Li LH, Zhang N, et al. A meta-analysis of the association of STAT4 polymorphism with systemic lupus erythematosus. Mod Rheumatol. 2010;20(3):257-62.

[3] Gupta V, Kumar S, Pratap A, Singh R, Kumari R, Kumar S, et al. Association of ITGAM, TNFSF4, TNFAIP3 and STAT4 gene polymorphisms with risk of systemic lupus erythematosus in a North Indian population. Lupus. 2018;27(12):1973-9.

Disclosure of Interests: None declared

DOI: 10.1136/annrheumdis-2021-eular.3470

\section{AB0014 1 METHOTREXATE - IMPLICATIONS OF PHARMACOGENETICS IN THE TREATMENT OF PATIENTS WITH RHEUMATOID ARTHRITIS}

R. Pinheiro Torres ${ }^{1,2}$, F. Pimentel Dos Santos ${ }^{1,2,3}$, J. Branco ${ }^{1,2,3} \cdot{ }^{1}$ Hospital Egas Moniz, Rheumatology, Lisboa, Portugal; ${ }^{2}$ CEDOC, Rheumatology, Lisboa, Portugal; ${ }^{3}$ Nova Medical School, Rheumatology, Lisboa, Portugal

Background: Methotrexate (MTX) is an anti-folate drug with anti-proliferative and anti-inflammatory effects. MTX proved to be the most highly effective, fast-acting disease modifying anti-rheumatic drug (DMARD), being widely used for the treatment of rheumatoid arthritis (RA) (1).

Objectives: This review aims to describe the main genetic variants identified concerning proteins that play a role in methotrexate's kinetics and efficiency profile.

Methods: A literature review was conducted since January of 2000 until December 2020, by searching the PubMed and Embase bibliographic databases, employing the following MeSH terms: methotrexate, pharmacogenetics, pharmacokinetics and rheumatoid arthritis. The search was limited to articles in English language. Two independent reviewers screened the titles and abstracts followed by a full-text review to assess papers regarding their eligibility. A total of 48 articles matched the research criteria and were analysed.

Results: Genetic variants of four main proteins, with different functions, have been consistently described.

Reduced folate carrier 1 (RFC1), a constitutively expressed folate transport protein that has high affinity for MTX is responsible, almost exclusively, for the transport of folate and MTX into the cell. The most commonly studied variant of the gene is the $80 \mathrm{G}>\mathrm{A}$ variant ( $r$ 1051266), mapped within exon 2, on chromosome 21. It seems to improve RA responses to MTX, clinical efficacy with long disease remission (2). 